\title{
Physicochemical investigation of chemical paint removers II: Role and mechanism of phenol in the removal of polyurethane coatings
}

\author{
Christopher N. Young*, Clive R. Clayton \\ Department of Materials Science and Engineering, Stony Brook University, Stony Brook, NY \\ 11794 \\ James H. Wynne, James P. Yesinowski, Grant C. Daniels \\ Chemistry Division, U.S. Naval Research Laboratory, 4555 Overlook Ave SW, Washington, DC \\ 20375
}

Purpose: Commercial military paint stripper is effective because it contains phenol; in order to replace it, one must first understand the mechanism of action/interaction with the coating. Target Journal: Progress in Organic Coatings (Elsevier)

Keywords: Paint stripping; methylene chloride; phenol; spectroscopy; polyurethane

*Christopher N. Young may be reached at: Christopher.young@alumni.stonybrook.edu

\begin{abstract}
Thermal and spectroscopic techniques have been used to study the interactions of phenol with model polyurethane coatings. Previous work has pointed to phenol as the principal source of polymer degradation in methylene chloride / phenol paint stripping mixtures. Thermal analysis demonstrates that the addition of phenol leads to severe depression in $\mathrm{T}_{g}$ and outright coating failure. Exposure appears to cause chain scission, leading to significant weight loss during thermal analysis. XPS confirms the deposition of methylcellulose stabilizer at the surface, but also indicates breaches in the conformal coating which we attribute to polyurethane degradation. Raman and ATR-FTIR spectroscopy confirm chemical modification of the polyurethane by phenol, leading to the proposed model of degradation by nucleophilic attack.
\end{abstract}

\section{Introduction}

The current formulation of chemical paint removers based on methylene chloride and phenol has proven extremely effective in the removal of polymeric coatings. These solvents also present significant challenges in modern use due to their adverse impacts on health and the environment. Recent regulations have elevated the urgency for less-hazardous paint removal techniques. While media blasting and laser ablation accomplish this objective, these methods are 
slow and require significant (expensive) retooling of current operations. Solvent-based methods currently rely on reformulation to replace methylene chloride and phenol with benzyl alcohol and anisole [1]. This mixture requires an order of magnitude more time to perform adequate paint removal (several hours $v s$. minutes). In order to determine optimal replacement solvents, one must first understand the mechanism of the target compound.

These authors have previously discussed the changes caused by the presence of methylene chloride (MC) in model military top-coatings [2]. In short, it was found that methylene chloride appears to swell the polyurethane polymer, evidently by solvating the carbonyl groups responsible for cross-linkage. This solvation effect is demonstrated through observation of Raman broadening of the carbonyl stretching peak, through the reduced rotational mobility of methylene chloride within the swollen polymer by both ${ }^{1} \mathrm{H}$ and ${ }^{2} \mathrm{H}$ NMR (of the deuterated methylene chloride molecule), and by increased polymer segmental motion by ${ }^{1} \mathrm{H}$ NMR. However, while methylene chloride can be observed to swell the coating during exposure, there is only a minimal subsequent change in $\mathrm{T}_{g}$ after drying; drying and swelling relaxation appear to occur within minutes of the cessation of solvent exposure. When one considers the nature of the effects of methylene chloride in combination with ethanol and water, along with the previous assessment of the active ingredients of these paint removers (see Introduction section of [2]), it is logical to hypothesize that the addition of phenol is primarily responsible for significant irreversible degradation in the polymeric coating.

Phenol is a relatively small alcohol that has demonstrated a strong ability to penetrate organic coatings and materials [3]. In addition to its penetrative characteristics, phenol offers numerous other advantages as a component of paint strippers. It is soluble in water, ethanol, and methylene chloride, and is capable of forming hydrogen bonds due to electron delocalization 
from the phenyl ring onto the oxygen atom. This delocalization also makes phenol a weak organic acid, which facilitates possible nucleophilic attack. Despite this acidity, no corrosive effects have been observed due to phenol, which leads to its suitability in aerospace applications. It has been speculated that phenol serves as a co-solvent to enhance the functionality of the primary solvent (here, methylene chloride) [4].

Recent work by Croll has presented solvent-based paint removal from a mechanical disbondment perspective, with the Flory-Rehner equation and Griffith fracture criteria used to interpret removal from the perspective of the cohesive and adhesive properties of the polymer [5]. Others have described the role of substrate receptor group density on adhesion [6-8], lending a chemical underpinning to disbondment. While these findings offer an explanation for removal, they do not address the chemical processes which may occur within the coating during solvent exposure, thereby leading to apparent chemical degradation. Extensive studies have been undertaken concerning the degradation of military coatings by water [9], accelerated weathering [10-15], and physical aging [16]. While polyurethanes are commonly considered susceptible to phenol exposure, the specific mechanism of attack has not been elucidated.

In this study, we discuss the effects of phenol in combination with other model formulation components on model military coatings, in order to elucidate the mechanism that allows phenol to cause irreversible damage to the coating. Model paint-removing systems, based upon those currently in service, have been constructed to permit analysis of solvent-polymer interactions with a minimum of interference. Differential Scanning Calorimetry (DSC) and Thermogravimetric Analysis (TGA) were used to monitor the change in polymer thermal properties. Vibrational spectroscopy techniques (specifically FTIR and Raman) were used to interrogate the chemical changes in the coating following solvent exposure. 


\section{Materials and Methods}

\subsection{Chemicals and Coatings}

All chemicals were reagent grade and used without further purification. Mixtures were prepared by weight percent according with Table 1 .

\begin{tabular}{|l|l|l|l|l|l|l|}
\hline Solution & Compound & $\begin{array}{l}\text { Methylene } \\
\text { Chloride }\end{array}$ & Ethanol & Water & Phenol & Methocel \\
\hline & Commercial paint stripper & & & & & \\
& 60.6 & 5.8 & 7.8 & 15.8 & 1.2 \\
\hline $\mathrm{D}$ & $\mathrm{MC}, \mathrm{EtOH}, \mathrm{H}_{2} \mathrm{O}, \mathrm{PhOH}$ & 67 & 6 & 9 & 18 & 1.2 \\
\hline $\mathrm{E}$ & $\mathrm{EtOH}, \mathrm{PhOH}$ & 0 & 27 & 0 & 73 & 0 \\
\hline $\mathrm{F}$ & $\mathrm{MC}, \mathrm{PhOH}$ & 79 & 0 & 0 & 21 & 0 \\
\hline
\end{tabular}

Table 1: List of control formulations by weight percent.

$a$ Contains also, Toluene (1.3\%), sodium petroleum sulfonate (5.5\%) and paraffin wax (1.9\%). Methocel is used to enhance solvent phase miscibility and to increase viscosity.

Current military coatings were selected for study, in the form of two polyurethane topcoats, based upon military specifications MIL-DTL-53039 (coating PU-1, an aliphatic isocyanate-based polyurethane with polyester extenders) and MIL-PRF-85285 (PU-2, a polyisocyanate-based polyurethane with polyether extenders); these coatings are designed to meet the referenced specifications, so their exact formulation may not be described in further detail. The coatings were unsupported free films, with a final thickness of approximately 5 mils (see Table 2). The simplification of the otherwise complex coating system was prepared to allow for ease of analysis. Coatings used in this study were clear films, without pigments, fillers and pigment related dispersion and wetting agents. Antifoaming agents were retained to facilitate sample preparation. Current formulations were used based on the same raw materials and binder ratios used in the commercial product. However, the solvent ratios were altered to compensate for the change in surface tension, viscosity and other properties resulting from the removal of the pigments and other components. Initial attempts at applications without this alteration of solvent 


\begin{tabular}{|r|c|c|c|c|}
\hline Coating & Coating Type & $\begin{array}{c}\text { Military } \\
\text { Specification }\end{array}$ & $\begin{array}{c}\text { Final thickness } \\
(\mathrm{mm})\end{array}$ & Specific coating description \\
\hline PU-1 & Polyurethane & MIL-DTL-53039 & 0.13 & $\begin{array}{c}\text { single component aliphatic polyurethane } \\
\text { CARC topcoat }\end{array}$ \\
\hline PU-2 & Polyurethane & MIL-PRF-85285 & 0.13 & $\begin{array}{c}\text { two component high solids polyurethane } \\
\text { topcoat }\end{array}$ \\
\hline
\end{tabular}

Table 2: List and description of prepared coatings.

\subsection{Sample Exposure}

Approximately two centimeter squares of each free coating film were cut and placed into individual scintillation vials. To each vial the respective solvent or solvent mixture was added until the film was completely covered $(\sim 10 \mathrm{~mL})$. After exposure, the liquid was decanted, the surface of the film rinsed with $5 \mathrm{~mL}$ EtOH to remove any impurities that may have been extracted and remained on the surface, and the film allowed to air dry in the open vial for $>2$ weeks. Samples were visually observed to be dry prior to analysis. DSC, TGA, Raman, FTIR, and XPS experiments were performed on samples exposed for $2 \mathrm{~h}$, with an additional set of samples exposed for 2 days analyzed by DSC. 


\subsection{Differential Scanning Calorimetry (DSC)}

Differential scanning calorimetry (DSC) was performed on a TA Instruments Q20 DSC with the DSC Refrigerated Cooling System (RCS) and a purge gas of nitrogen set to $50 \mathrm{~mL} / \mathrm{min}$. Samples of approximately 1-2 mg were placed into TA Instruments Tzero aluminum pans, with an empty aluminum pan used as reference. Samples were analyzed twice from $-90{ }^{\circ} \mathrm{C}$ to $150{ }^{\circ} \mathrm{C}$ at a heating rate of $20{ }^{\circ} \mathrm{C} / \mathrm{min}$. All data reported were taken from the second scan in order to minimize the effect of shape memory. Glass transition temperatures were determined using TA Universal Analysis program.

\subsection{Thermogravimetric Analysis (TGA)}

Thermogravimetric analysis (TGA) was done on a TA Instruments Q50 TGA using a platinum sample pan. The analysis was carried out with air used as the sample purge gas. Nitrogen was used as the purge gas for the balance. Data were recorded from ambient temperature to $700{ }^{\circ} \mathrm{C}$ at $5{ }^{\circ} \mathrm{C} / \mathrm{min}$. Plots of percent weight loss versus temperature were made to analyze the data.

\subsection{FTIR-ATR}

FTIR spectra were recorded on a Thermo Scientific Nicolet 6700 FTIR spectrometer equipped with a Smart Performer ATR attachment using a germanium crystal and constant pressure tower, and also with a Smart iTR attachment using a zinc selenide crystal and constant pressure tower. A DTGS detector collected 64 scans of the sample. Data acquisition and analysis was performed using the Thermo Scientific OMNIC software suite. 


\subsection{Raman}

Raman analysis was carried out using a Nicolet Almega dispersive spectrometer coupled to an Olympus microscope with 10x and 100x objective lenses, and also using a WiTec $\alpha-500$ confocal Raman microspectrometer with 20x and 100x objective lenses. The sample was scanned 128 times using a 1s collection time, with the spectra analyzed using the Thermo Scientific OMNIC software suite. Samples were placed on quartz slides to reduce background interference. In the case of the solvated coatings, samples were placed on a quartz slide with additional solvent, then covered with a second quartz slide to maintain sample geometry and diffuse solvent evaporation away from the objective. Additional solvated samples were analyzed by adhering the sample to the inner face of a quartz cuvette, which was then capped and placed horizontally for analysis. Efforts were made to minimize sample heating while performing analysis.

\subsection{Entrapped Vapor and Supernatant GC-MS}

Vapor above a free coating film (headspace) in an air tight vial was injected into the GCMS after the vial had been heated at approximately $80^{\circ} \mathrm{C}$ for 30 minutes. The GCMS system was an Agilent 7890A gas chromatograph equipped with an Agilent 5975C mass selective detector operating in electron ionization mode and an Agilent 7693A autoinjector. The column utilized was an Agilent HP-5MS (5\% phenyl) methylpolysiloxane film. The carrier gas was helium with a flow rate of $1 \mathrm{~mL} \cdot \mathrm{min}^{-1}$. The injection volume was $10 \mu \mathrm{L}$ with a split injection ratio of 50:1. The temperature program has an initial temperature of $35^{\circ} \mathrm{C}$ for one minute, then $10{ }^{\circ} \mathrm{C}$ per minute ramp to $90{ }^{\circ} \mathrm{C}$ with a three minute post run hold at $250{ }^{\circ} \mathrm{C}$. The injection port temperature, MS quad temperature, and source temperature were $220^{\circ} \mathrm{C}, 150{ }^{\circ} \mathrm{C}$ and $230{ }^{\circ} \mathrm{C}$, respectively. The detector was set to scan a mass range from 20 to $500 \mathrm{~m} / \mathrm{z}$. For analysis of the 
supernatant liquid after exposure of a free coating film, the initial oven temperature was $50{ }^{\circ} \mathrm{C}$ held for one minute, then ramped up to $300{ }^{\circ} \mathrm{C}$ at $15{ }^{\circ} \mathrm{C}$ per minute.

\subsection{XPS}

X-ray photoelectron spectra were obtained using a VG ESCA-3 Mk. II system under ultra-high vacuum $\left(10^{-9}\right.$ Torr $\left.\equiv 10^{-7} \mathrm{~Pa}\right) . \mathrm{X}$-rays were generated from a magnesium anode at 6 $\mathrm{kV}$ with a filament current of $20 \mathrm{~mA}$. A pass energy of $20 \mathrm{eV}$ was applied to emitted photoelectrons for analysis. Scans for each region were executed with a spacing of $0.1 \mathrm{eV}$ and a 100ms dwell time; each scan was repeated 50 times and co-added, using the VGX900i control program. Spectra were calibrated using the adventitious carbon peak at $284.5 \mathrm{eV}$. Analysis was performed using the CasaXPS software package.

\subsection{Microscopy}

Optical micrographs were obtained using an Olympus OLS4000 3D Measuring Laser Confocal Microscope. Scanning electron micrographs were obtained using an FEI LEO SEM with backscatter detector.

\section{Results and Discussion}

\subsection{Thermal properties}

In Table 3, the changes in $\mathrm{T}_{g}$ due to exposure to the prepared phenol solvent mixtures are shown. Phenol produces significant, irreversible deterioration of the coating observed through multiple technique including SEM and confocal microscopy, as highlighted in Figure 1. Its addition leads to $\mathrm{T}_{g}$ depressions of well over $50{ }^{\circ} \mathrm{C}$, which results in a soft, easily-torn film at room temperature. In the case of coating PU-2, the clear film became so swollen and soft that 
removal of the film from solution was impossible. One would expect that solvent system D would lead to decomposition in the fully-formulated coating as well, or at least lead to the greatest $\mathrm{T}_{g}$ depression. We explain this based on the nature of the coating: fully-formulated PU2 has a glass transition temperature of $0{ }^{\circ} \mathrm{C}$, indicating that it is highly flexible in the presence of various standard fillers and pigments used in the coating system, such as celite and titanium dioxide. We speculate that the encapsulation of these fillers within the polyurethane matrix serves to provide some minimal flexible cohesion which is unaffected by phenol; further research into the critical concentration of fillers is required. The application of solvent system $\mathrm{F}$ to free-standing films of fully-formulated PU-1 leads to significant degradation and apparent failure by shear force. While plasticization is occurring due to the presence of phenol, these effects are too significant to be accounted for solely by this mechanism; a later section will discuss the spectroscopic evidence for polymer degradation.

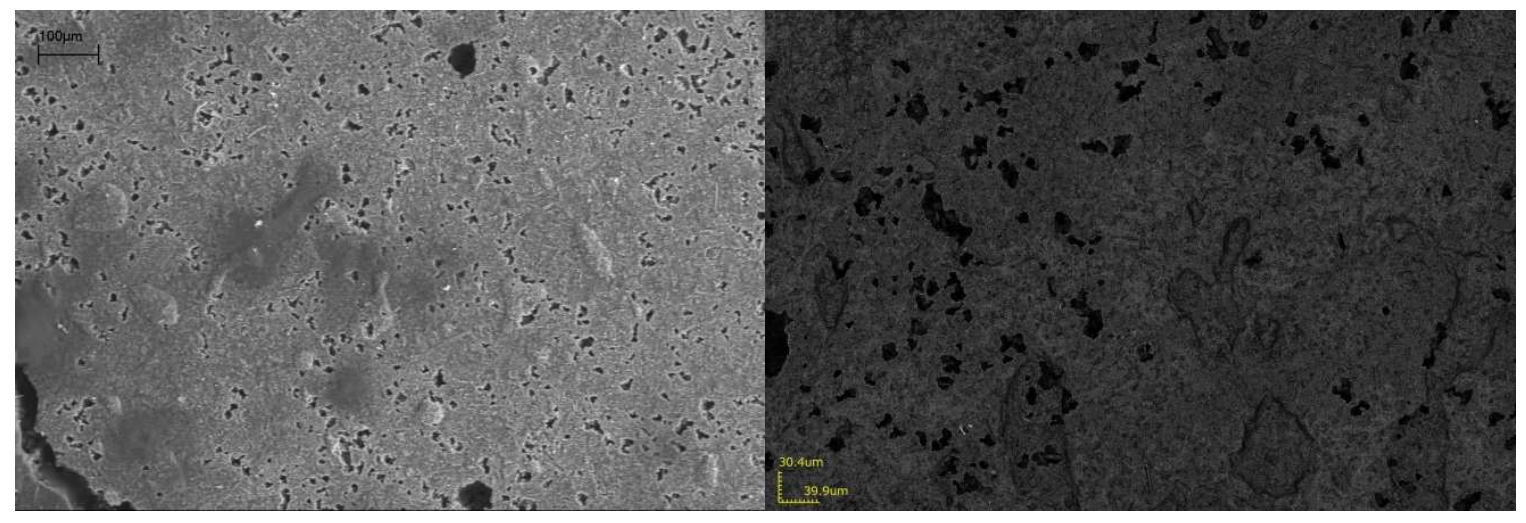

Figure 1 - SEM (left) and optical (right) micrographs of clear PU-1 exposed to solvent system D, highlighting the significant apparent damage to the coating. 


\begin{tabular}{|c|c|c|c|c|}
\hline & \multicolumn{2}{|c|}{ PU-1 Polyurethane Topcoat } & \multicolumn{2}{c|}{ PU-2 Polyurethane Topcoat } \\
\hline Solvent Exposure & $\begin{array}{c}\text { Clear } \\
\text { coating }\end{array}$ & $\begin{array}{c}\text { Fully formulated } \\
\text { coating }\end{array}$ & $\begin{array}{c}\text { Clear } \\
\text { coating }\end{array}$ & $\begin{array}{c}\text { Fully formulated } \\
\text { coating }\end{array}$ \\
\hline Control & 87 & 60 & 51 & 0 \\
\hline MC/EtOH/Water/PhOH* (D) & -11 & -8 & DECOMP & -38 \\
\hline EtOH/PhOH (E) & -9 & -13 & -25 & -45 \\
\hline MC/PhOH (F) & -4 & -2 & -30 & -54 \\
\hline
\end{tabular}

Table $3-\mathrm{T}_{\mathrm{g}}$ values in degrees Celsius for clear and fully-formulated polyurethane coatings exposed to phenolcontaining solutions.

* - Solvent mixture stabilized with Methocel

\subsection{Solvent persistence}

The exposed coatings acquire a light purple tint (shown in Figure 2) as a result of exposure, indicative of the presence of impure solid phenol within the polymer matrix. Coatings also produce a strong odor of phenol after several months of drying, indicating solvent retention. Small samples of exposed films were heated in sealed vials, and the subsequent headspace gas was analyzed using GC-MS, where the presence of phenol was confirmed.

Figure 2 - Clear coating PU-1 (MIL-53039) after exposure to solvent system D for $2 \mathrm{~h}$ with subsequent drying, showing a purple tint indicative of impure solid phenol.

Thermogravimetric analysis (TGA) was performed on all exposed coatings, with an unexposed coating serving as control. Curves are presented in Figure 2, with phenol-containing solvent results overlaid against those from solvent blends without phenol. Following exposure to solvent mixtures containing phenol, each coating shows a significant loss of weight before 
reaching $125^{\circ} \mathrm{C}$. The boiling point of phenol is well above this point $\left(181^{\circ} \mathrm{C}\right)$, which suggests that phenol is causing significant chain scission within the sample, with subsequent outgassing of low-molecular weight degradation products. The mass loss at $\sim 125^{\circ} \mathrm{C}$ is believed to be ultimately caused by chain scission and not entrapped water/solvent. Sublimation of phenol may be affecting the TGA curves of the sample; however, the intercalation and incorporation of the phenol would lead to much slower rate of sublimation due to hindered migration through the polymer matrix as compared to that of pure solid phenol. A mass loss of 50-60\% from the sample by $125^{\circ} \mathrm{C}$ indicates, along with other data provided, that degradation of the free film has occurred. This was concluded after head space analysis yielded no signs of organic solvent, yet the limits of detection of GPC were not sufficient to detect substantial molecular weight extracts or oligomers believed to be evolving from the thermal process. The smaller mass loss at $\sim 125$ ${ }^{\circ} \mathrm{C}$ for samples not exposed to phenol might be accounted for by migration of unreacted products or short-chain segments not cross-linked during curing. 

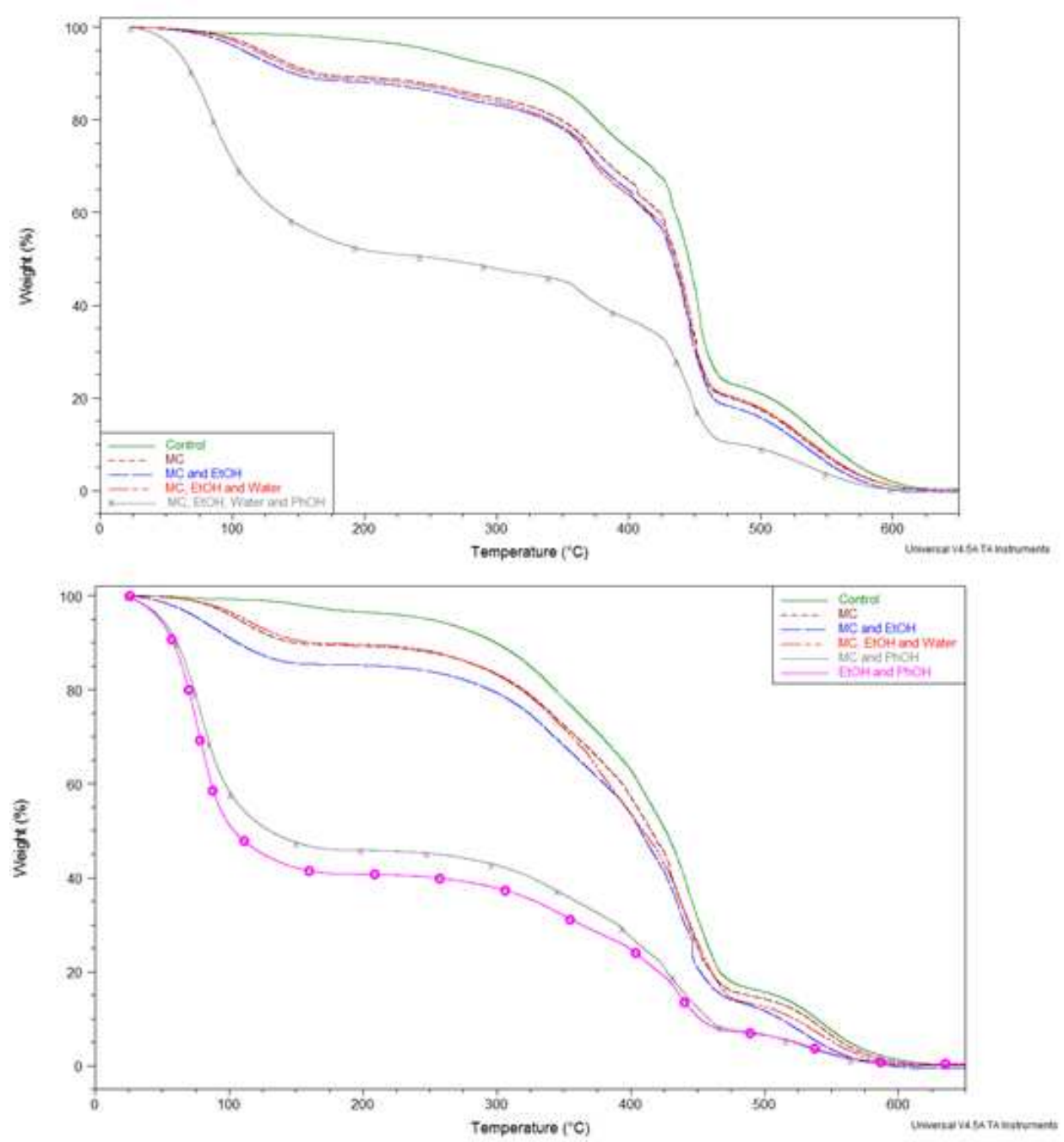

Figure 3 - TGA curves of coatings PU-1 (top) and PU-2 (bottom) exposed to solvent systems D, E, and F; as well as systems $\mathrm{A}, \mathrm{B}$, and C (100\% MC, 91\% MC / 9\% EtOH, and 82\% MC / 8\% EtOH / 10\% $\mathrm{H}_{2} \mathrm{O}$, respectively) from past work [2].

\subsection{Spectroscopy of exposed polyurethanes - solvent system D}

Polyurethane is observed to degrade significantly after exposure to phenol-containing solvent mixtures. In Figure 3, we present ATR-FTIR spectra of coating PU-1 exposed to mixture $\mathrm{D}$, which causes the greatest $\mathrm{T}_{g}$ depression in this system. There is spectral evidence of a significant residue of hydroxypropyl methylcellulose on the surface of the material; as mentioned in the previous paper, this masks many changes which occur in the coating. The spectrum demonstrates significant attenuation of the signal (or peak, or absorption) from the 
polyurethane binder, with the primary signatures coming from methylcellulose and phenol (most notably observed by the presence of C-O and C-O-C peaks between 1000 and $1250 \mathrm{~cm}^{-1}$, and $\mathrm{C}=\mathrm{C}$ at $\left.1596 \mathrm{~cm}^{-1}\right)$. It is difficult to evaluate chemical changes in these samples with such a shallow depth of analysis.

Also in Figure 3, the complementary spectrum from Raman analysis is presented. Herein, we see the similar evolution of C-O-C due to methylcellulose, in addition to the numerous persistent peaks due to phenol (such as those at 1595 and $1000 \mathrm{~cm}^{-1}$, indicating $\mathrm{C}=\mathrm{C}$ aromatic and aromatic ring breathing, respectively). The peak at $1000 \mathrm{~cm}^{-1}$ is caused by aromatic ring breathing, and its growth is attributable to the presence of phenol. The overall difficulty in this analysis arises because the most likely chemical changes in the coating are generally masked by similar bonds in methylcellulose and phenol - these common peaks are indicated in the FTIR spectrum.
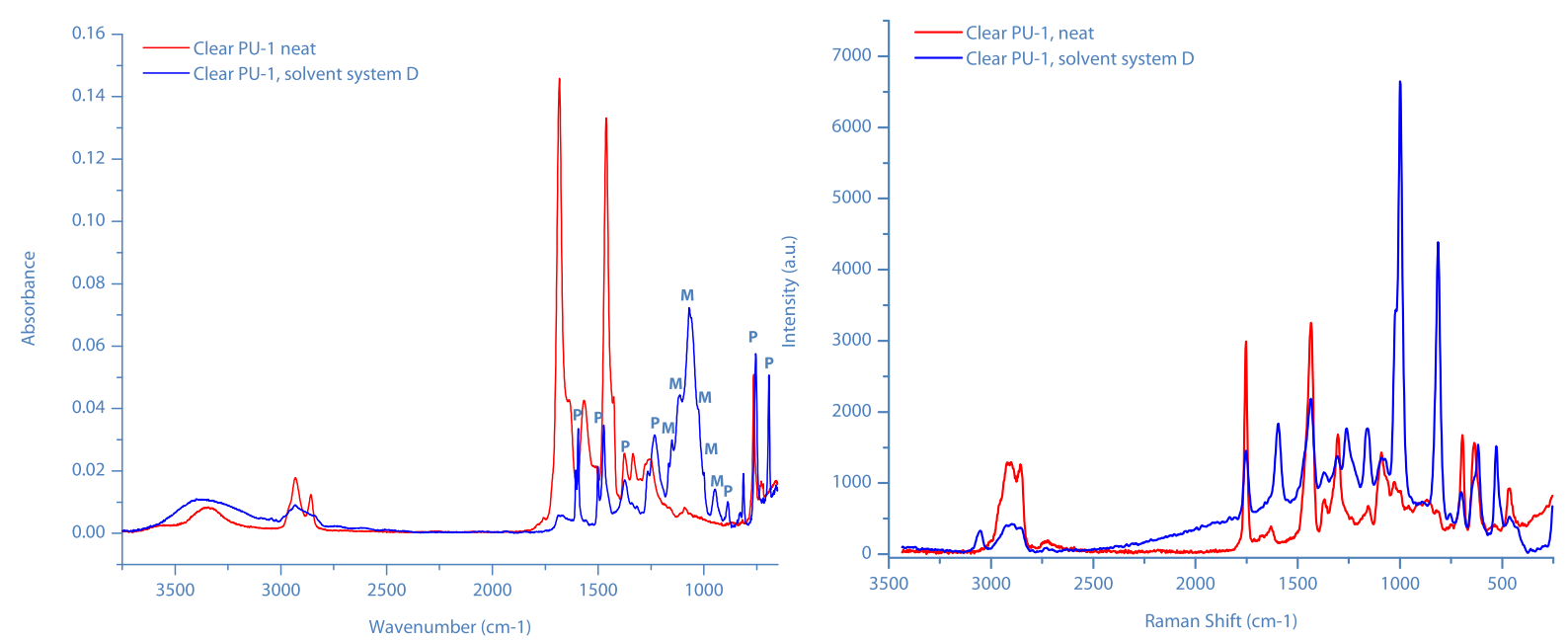

Figure 4 - (left) ATR-FTIR and (right) Raman spectra of clear coating PU-1 before and after exposure to solvent system D. In the FTIR spectrum, peaks which occur from phenol are labeled with $\mathbf{P}$, and peaks which occur from methylcellulose are labeled with $\mathbf{M}$. 

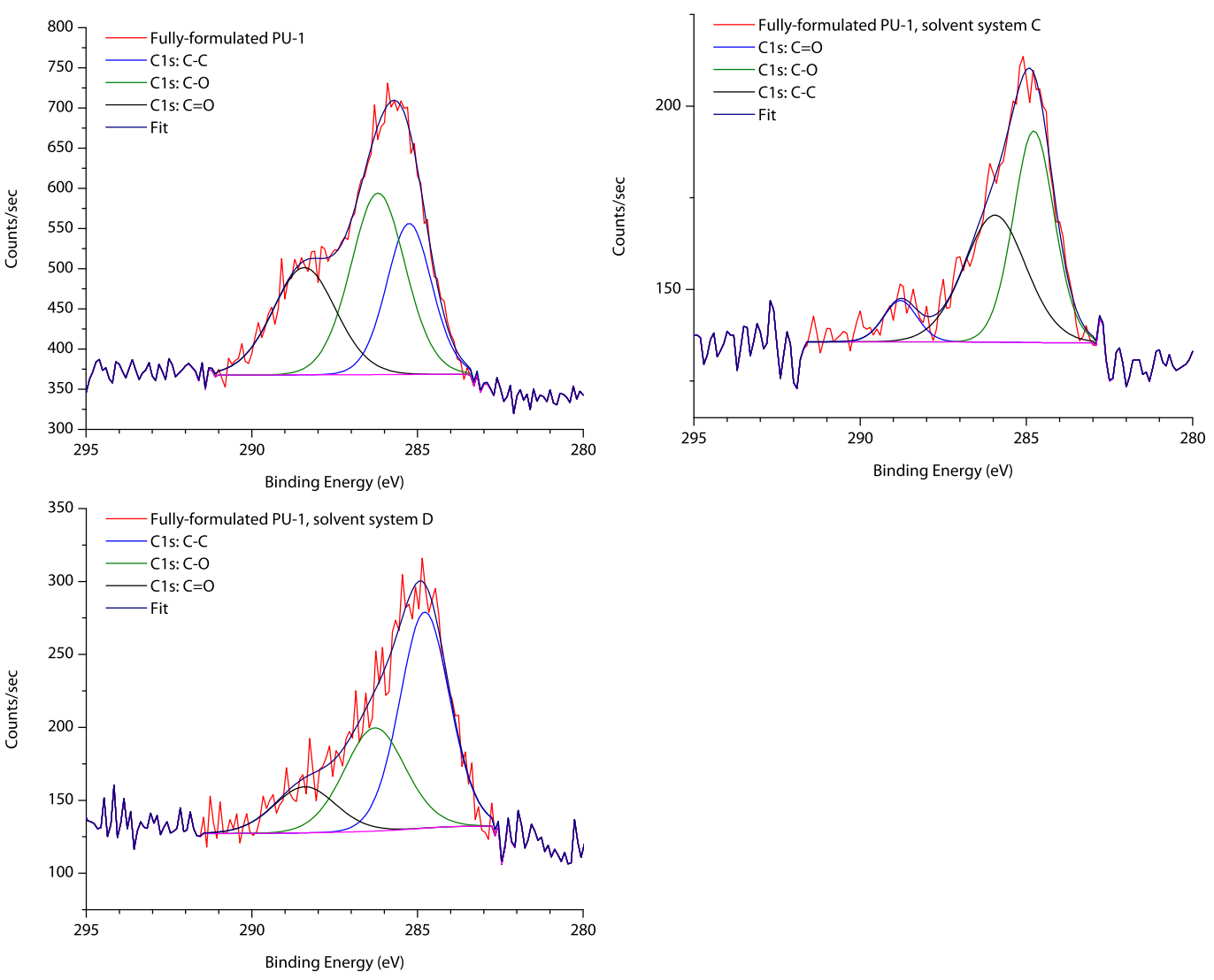

Figure 5 -XPS C1s spectra of fully-formulated PU-1 unexposed (top left), exposed to solvent system C (top right), and exposed to solvent system $D$ (below left). 


\subsection{Spectroscopy of exposed polyurethanes - solvent system $F$}

In order to isolate the effects of phenol, coatings were exposed to a solvent system without methylcellulose; while this system is marginally less effective than solvent system D, the interactions of phenol are likely to be similar in nature. The ATR-FTIR spectra of system PU-2 before and after exposure to solvent system $\mathrm{F}$ are shown in Figure 5; the preponderance of phenol in the sample does skew the peak intensities of the polymer, rendering quantitative analysis difficult. Several changes in the spectrum are immediately obvious. Prior to exposure, the larger peak at $1684 \mathrm{~cm}^{-1}$ is attributed to hydrogen-bonded urethane carbonyl [17], and the shoulder at $1728 \mathrm{~cm}^{-1}$ indicates ester carbonyl from the polyester-polyol extender [14]. The peak at $1645 \mathrm{~cm}^{-1}$ indicates hydrogen-bonded urea [18] (resulting from the polyisocyanate form of the precursor, hexamethylene diisocyanate, which is in the form of a biuret (i.e. $\left.-(\mathrm{HN}-\mathrm{CO}-)_{2} \mathrm{~N}-\right)$ for this application, and from the inevitable formation of urea as a result of isocyanate-water reactions) [19].

After exposure, the hydrogen-bonded urethane peak splits into two peaks at 1670 and $1687 \mathrm{~cm}^{-1}$. Based on the splitting pattern, we propose that the peak at $1687 \mathrm{~cm}^{-1}$ corresponds to hydrogen-bonded urethane carbonyl as before (and thus shifted to a small degree due to steric effects of the presence of phenol), and that the peak at $1670 \mathrm{~cm}^{-1}$ corresponds to a urethane carbonyl vibration near a chain end, of the form $\mathrm{NR}_{2}-\mathrm{CO}-\mathrm{O}-\mathrm{Ar}$ (resembling phenyl carbamate). The carbonyl position for phenyl carbamate occurs slightly above $1700 \mathrm{~cm}^{-1}$; given the usual pattern of roughly $20-25 \mathrm{~cm}^{-1}$ shift depending on the degree of hydrogen bonding [20], it is reasonable to suggest this position as a possible location.

A new shoulder is formed at $1265 \mathrm{~cm}^{-1}$; it is recognized that aryl monosubstitution increases the wavenumber in asymmetric C-O-C stretching vibrations [21], thus further 
supporting the phenyl carbamate model. The amide II peak centered at $1540 \mathrm{~cm}^{-1}$ is significantly decreased, and reduces into two peaks at 1530 and $1555 \mathrm{~cm}^{-1}$, which might plausibly be attributed to the hydrogen bonding in dilute solution [21]. The DSC data presented above supports this notion, as the strongly depressed $\mathrm{T}_{g}$ value indicated in Table 3 suggests some measure of polymer chain cleavage to so severely impact the material. GC-MS of the supernatant for films exposed to solvent system F revealed a shoulder peak on phenol indicative of carbamic acid, methyl-, phenyl ester. While small, the peak for carbamic acid supports the notion that this change occurs as a result of nucleophilic attack, as explained schematically in Figure 7, due to the weak acidity of the phenol molecule. The peaks which correspond to O-H stretching around $3370-3500 \mathrm{~cm}^{-1}$ do increase in intensity, but this increase is also influenced by the presence of unbound phenol, as noted through GC/MS headspace analysis discussed earlier. Generally, the presence of both residual phenol and existing esters masks many changes we would otherwise hope to detect in the coating, but the observation of urethane carbonyl peak splitting and the development of a new C-O-C shoulder both support this reaction mechanism. By eliminating the presence of methylcellulose, we confirm that these observed changes are caused by a chemical reaction, rather than cellulosic deposition at the surface as seen with stabilized solvent blends. 


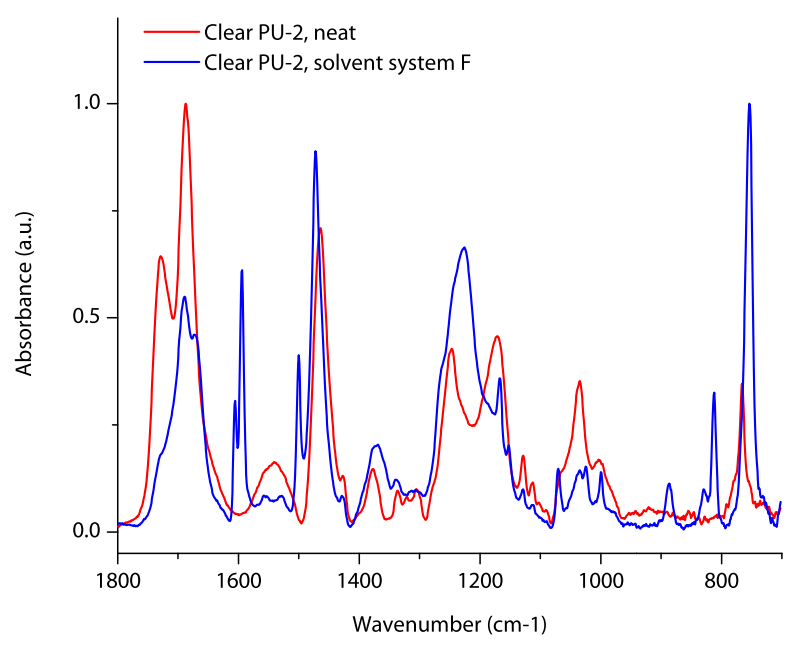

Figure 6 - (left) ATR-FTIR spectra of PU-2 before and after exposure to solvent system F; (right) a magnified view of the fingerprint region of these spectra.

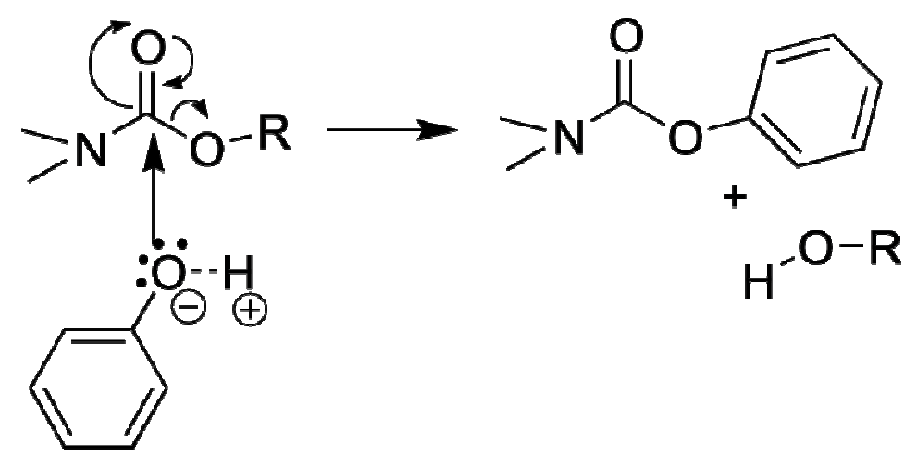

Figure 7 - Proposed nucleophilic attack of phenol on polyurethane.

A comparable effect is observable in coating PU-1, presented in Figure 7. The carbonyl peak at $1683 \mathrm{~cm}^{-1}$ is split into two peaks at 1687 and $1670 \mathrm{~cm}^{-1}$, suggesting the same mechanism seen in PU-2. It is more straightforward to resolve the shoulder at $1265 \mathrm{~cm}^{-1}$ in this sample, as it is not crowded by the presence of other $\mathrm{C}-\mathrm{O}-\mathrm{C}$ polyester peaks due to the extender polymer chosen (here, usually polyethylene or a derivative), although the presence of phenol does still generate several peaks nearby. From the thermal data, we know that the degradative performance of phenol is enhanced by the addition of a small amount of water. This seems likely to be due to the enhanced acidity of phenol in the presence of water. The Raman spectrum 
of this exposure (Figure 8) is dominated by the presence of phenol, and since it is not as sensitive as FTIR to the changes observed in this degradation, it does not shed any additional light on the situation.
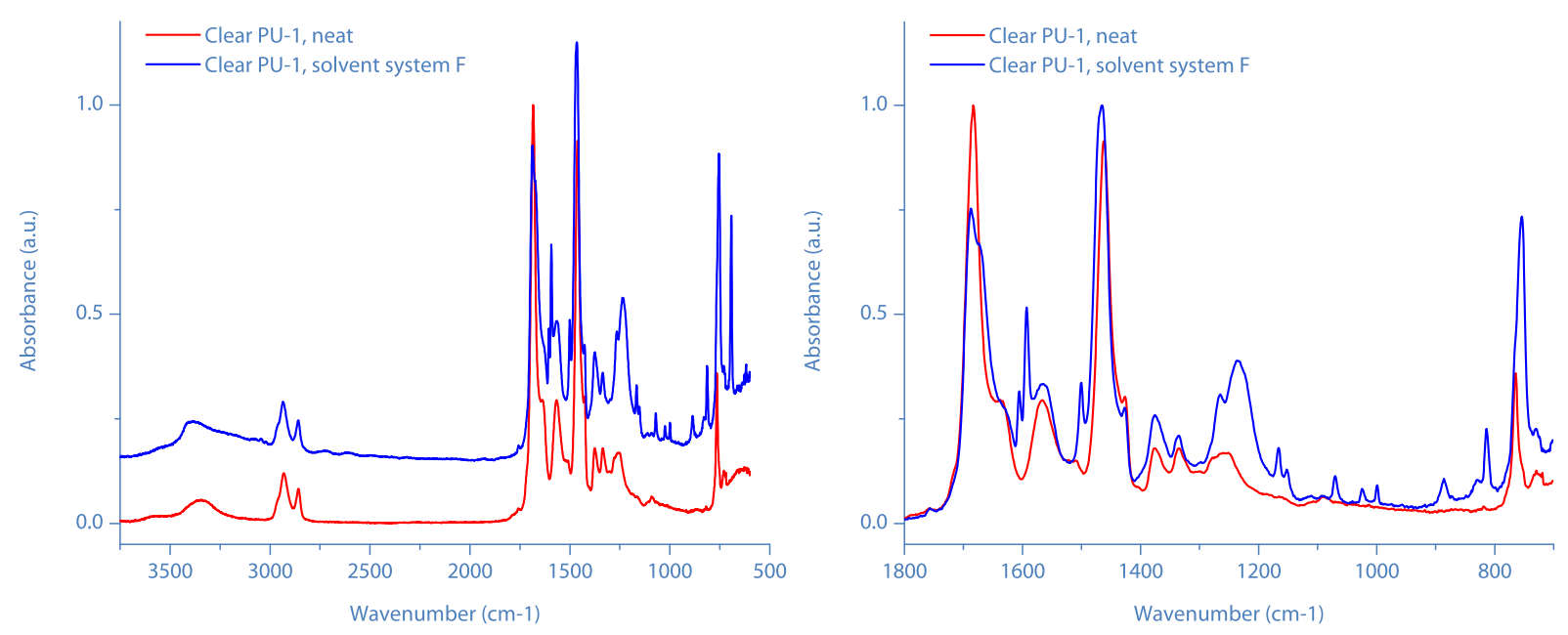

Figure 8 - (left) ATR-FTIR spectra of PU-1 before and after exposure to solvent system F for 2h; (right) a magnified view of the fingerprint region of these spectra 


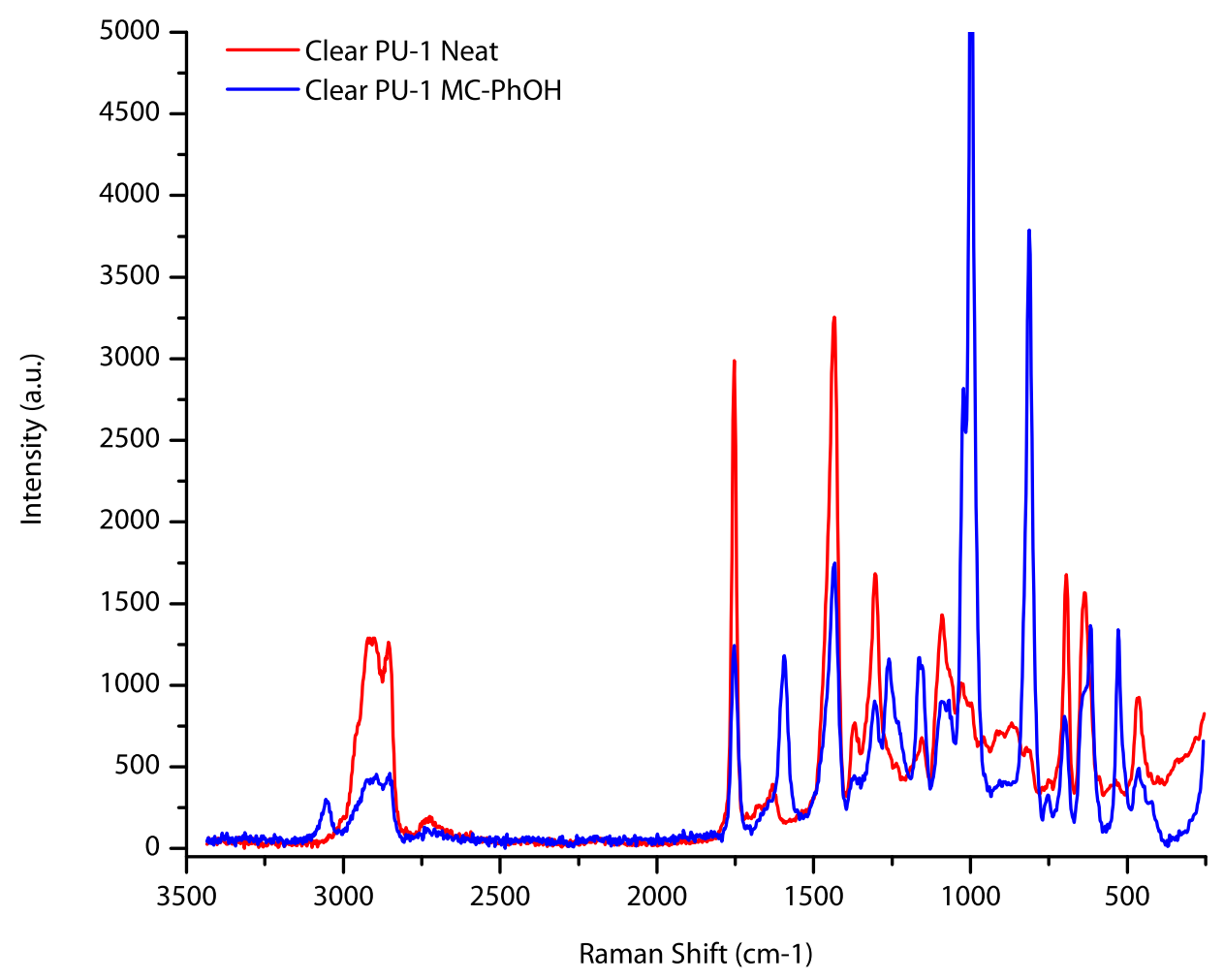

Figure 9-Raman spectra of clear PU-1 before and after exposure to solvent system F.

\section{Conclusions}

The addition of phenol to a model paint stripping solution results in significant, irreversible depression of $\mathrm{T}_{g}$ in exposed military coating systems. Phenol is retained in the sample for months after exposure, and can be detected by GC-MS analysis and a simple odor test. There is significant mass loss from the exposed coating at a much lower elevated temperature than can simply be accounted for by phenol evacuation, suggesting that a chemical reaction by phenol is resulting in chain scission and subsequent removal of the shortened chains. ATR-FTIR spectroscopy highlights a change in the degree and type of carbonyl/amine hydrogen bonding of the coating, as well as the development of a new aromatic ether peak, which allows 
us to suggest a model for degradation based on nucleophilic attack of the urethane linkage by the weakly-acidic phenol molecule.

\section{Acknowledgements}

The authors gratefully acknowledge Dr. John Kelley of the U.S. Army Research Laboratory (Aberdeen, MD) for coatings formulation expertise and for sample preparation. The authors acknowledge funding support from SERDP projects WP-1682, in conjunction with principal investigator Dr. Young Han of the U.S. Naval Air Systems Command (Patuxent River, MD), and WP-2244. Research carried out in part at the Center for Functional Nanomaterials, Brookhaven National Laboratory, which is supported by the U.S. Department of Energy, Office of Basic Energy Sciences, under Contract No. DE-AC02-98CH10886.

\section{References}

[1] DoD, Remover, Paint, No Hazardous Air Pollutants (HAPS), in, 2001.

[2] C.N. Young, C.R. Clayton, J.P. Yesinowski, J.H. Wynne, K.E. Watson, Physicochemical investigation of chemical paint removers: Interactions of methylene chloride with polyurethane coatings, Progress in Organic Coatings, 77 (2014) 232-241.

[3] K. Bjur, A. Cassel, I. Uneback, A. Stavtsov, Mercerizing an ionizing-radiated alpha-cellulose with alkali, followed by xanthating and dissolving the xanthated alpha-cellulose in an aqueous alkali solution; adding a nonionc and/or a cationic surfactant; byproduct inhibition; quality, in, Google Patents, 2000.

[4] T. Wollbrinck, The Composition of Proprietary Paint Strippers, Journal of the American Institute for Conservation, 32 (1993) 43-57.

[5] S.G. Croll, Application of the Flory-Rehner equation and the Griffith fracture criterion to paint stripping, J. Coat. Technol. Res., 7 (2010) 49-55.

[6] I. Lee, R.P. Wool, Polymer Adhesion vs. Substrate Receptor Group Density, Macromolecules, 33 (2000) 2680-2687.

[7] S.J. Hinder, J.F. Watts, C. Lowe, Interface analysis and compositional depth profiling by XPS of polymer coatings prepared using ultralow-angle microtomy, Surf. Interface Anal., 36 (2004) 1032-1036.

[8] S.J. Hinder, C. Lowe, J.T. Maxted, C. Perruchot, J.F. Watts, Intercoat adhesion failure in a multilayer organic coating system: An X-ray photoelectron spectroscopy study, Progress in Organic Coatings, 54 (2005) 20-27. 
[9] X.F. Yang, D.E. Tallman, S.G. Croll, G.P. Bierwagen, Morphological changes in polyurethane coatings on exposure to water, Polymer Degradation and Stability, 77 (2002) 391396.

[10] S.G. Croll, A.D. Skaja, Quantitative spectroscopy to determine the effects of photodegradation on a model polyester-urethane coating, Journal of Coatings Technology, 75 (2003) 85-94.

[11] X.F. Yang, J. Li, S.G. Croll, D.E. Tallman, G.P. Bierwagen, Degradation of low gloss polyurethane aircraft coatings under UV and prohesion alternating exposures, Polymer Degradation and Stability, 80 (2003) 51-58.

[12] X.F. Yang, D.E. Tallman, G.P. Bierwagen, S.G. Croll, S. Rohlik, Blistering and degradation of polyurethane coatings under different accelerated weathering tests, Polymer Degradation and Stability, 77 (2002) 103-109.

[13] X.F. Yang, C. Vang, D.E. Tallman, G.P. Bierwagen, S.G. Croll, S. Rohlik, Weathering degradation of a polyurethane coating, Polymer Degradation and Stability, 74 (2001) 341-351. [14] L.T. Keene, G.P. Halada, C.R. Clayton, Failure of navy coating systems 1: chemical depth profiling of artificially and naturally weathered high-solids aliphatic poly(ester-urethane) military coating systems, Progress in Organic Coatings, 52 (2005) 173-186. [15] L.T. Keene, M.J. Vasquez, C.R. Clayton, G.P. Halada, Failure of navy coating systems 2: failure pathways of artificially weathered navy coating systems applied to chromate conversion coated AA2024-T3 substrates, Progress in Organic Coatings, 52 (2005) 187-195.

[16] S.G. Croll, X. Shi, B.M.D. Fernando, The interplay of physical aging and degradation during weathering for two crosslinked coatings, Progress in Organic Coatings, 61 (2008) 136144.

[17] M.M. Coleman, D.J. Skrovanek, J. Hu, P.C. Painter, Hydrogen bonding in polymer blends. 1. FTIR studies of urethane-ether blends, Macromolecules, 21 (1988) 59-65.

[18] A. Marcos-Fernández, A.E. Lozano, L. González, A. Rodríguez, Hydrogen Bonding in Copoly(ether-urea)s and Its Relationship with the Physical Properties, Macromolecules, 30 (1997) 3584-3592.

[19] B. MaterialScience, The Chemistry of Polyurethane Coatings: A General Reference Manual, in: B. MaterialScience (Ed.), Bayer, Pittsburgh, PA, 2005.

[20] L. Irusta, J.J. Iruin, M.J. Fernández-Berridi, M. Sobkowiak, P.C. Painter, M.M. Coleman, Infrared spectroscopic studies of the self-association of ethyl urethane, Vibrational Spectroscopy, 23 (2000) 187-197.

[21] G. Socrates, Infrared Characteristic Group Frequencies: Tables and Charts, 2nd ed., John Wiley \& Sons, Chichester, 1994. 